\title{
Knowledge, attitude and practices about menstruation and related problems in adolescent girls
}

\author{
Shabana Sultan*, Deep Shikha Sahu
}

Department of Obstetrics and Gynecology, GMC, Bhopal, Madhya Pradesh, India

Received: 06 October 2017

Revised: 15 October 2017

Accepted: 28 October 2017

\author{
*Correspondence: \\ Dr. Shabana Sultan, \\ E-mail: drshabanasultan@gmail.com
}

Copyright: () the author(s), publisher and licensee Medip Academy. This is an open-access article distributed under the terms of the Creative Commons Attribution Non-Commercial License, which permits unrestricted non-commercial use, distribution, and reproduction in any medium, provided the original work is properly cited.

\begin{abstract}
Background: Menstruation and menstrual practices are still clouded by taboos and socio-cultural restrictions resulting in adolescent girls remaining ignorant of the scientific facts and hygienic health practices, which sometimes result into adverse health outcomes. The reaction to menstruation depends upon awareness and knowledge about the subject. The manner in which a girl learns about menstruation and its associated changes may have an impact on her response to the event of menarche. Department of Obstetrics and Gynecology, Bhopal, India had always been keen to make aware of these facts and preventive measures to adolescent girls and to acknowledge the facts this study has been conducted.

Methods: About 350 school girls between 10-18years of age with attainment of menarche were selected from two schools of Bhopal and interviewed during study period using predesigned questionnaire after obtaining informed consent from school in-charge and parents.

Results: This study shows that majority of the girls preferred cloth pieces rather than sanitary pads as menstrual absorbent. Apparently, poverty, high cost of disposable sanitary pads and to some extent ignorance dissuaded the study population from using the menstrual absorbents available in the market. Universalized use of sanitary pads can be advocated to every girl only by making it available at affordable prices (social marketing). Thus, the above findings reinforce the need to encourage safe and hygienic practices among the adolescent girls and bring them out of traditional beliefs, misconceptions and restrictions regarding menstruation.

Conclusions: This study highlighted the need of adolescent girls to have accurate and adequate information about menstruation and its appropriate management and healthy menstrual practices.
\end{abstract}

Keywords: Adolescent girls, Menarche, Menstruation, Menstrual absorbents, Menstrual hygiene, Sanitary pad

\section{INTRODUCTION}

According to United Nations Children's Fund (UNICEF), there are 243 million adolescents comprising $20 \%$ of the total population of India which clearly shows that India is truly "young". This sheer number itself is a big challenge in itself; 15-19 years constitute $10 \%$ and majority lives in rural areas. ${ }^{1}$ Adolescence is a period of rapid physical, emotional and psychological development and a period of adjustment with family and society. It is a phase of transition from girlhood to womanhood. Although, menstruation is a normal physiological phenomenon for females, it is often associated with some degree of sufferings and embarrassment. ${ }^{1,2}$

The first menstruation is often horrifying and traumatic to an adolescent girl because it usually occurs without her knowing about it. There is a substantial lacunae in the 
knowledge about menstruation among adolescent girls. Several traditional norms and beliefs influence the practices related to menstruation. It is a common observation that girls are rarely informed about menstruation unless they experience it for the first time. ${ }^{2}$

Many studies have revealed that most of the adolescent girls had incomplete and inaccurate information about the menstrual physiology and hygiene. It also revealed that mothers, television, friends, teachers and relatives were the main sources which provided information on menstruation to the adolescent girls. ${ }^{3,4}$ Hygiene-related practices of women during menstruation are of considerable importance, as it has a health impact in terms of increased vulnerability to reproductive tract infections (RTI).

The interplay of socio-economic status, menstrual hygiene practices and RTI are noticeable. Today millions of women are sufferers of RTI and its complications and often the infection is transmitted to the offspring of the pregnant mother. ${ }^{2}$

Women having better knowledge regarding menstrual hygiene and safe practices are less vulnerable to RTI and its consequences. Therefore, increased knowledge about menstruation right from childhood may escalate safe practices and may help in mitigating the suffering of millions of women.

Good hygienic practices such as the use of sanitary pads and adequate washing of the genital area are essential during menstruation. Women and girls of the reproductive age need access to clean and soft, absorbent sanitary products which can in the long run, protect their health. Menstrual hygiene and management will directly contribute to (MDG)-2 on universal education, MDG-3 on gender equality and women empowerment. ${ }^{5,6}$

Therefore, this study is done under four sections:

- Knowledge and attitude

- Problems during menstruation

- Seclusion and absenteeism

- Hygiene related practices

Objectives of present study were to study the knowledge and attitude of menstruation, to study the practices followed about menstrual hygiene and to study the various problems related to menstruation.

\section{METHODS}

This study was a Community-based cross-sectional observational study. The present study was undertaken among the 350 adolescent school girls in the field practice of 2 schools in Bhopal by department of obstetrics and gynecology Bhopal, India.
The study was carried out for Two month, July 2016August 2016. Three hundred and fifty girls of age between 10-18yrs but maximum were of age 14-16 years. Study tool was a pre-designed, pre-tested questionnaire.

After taking permission from the school authority, the class teachers were explained the purpose of the study and verbal consent was obtained from them. Briefing was done to the students regarding the questionnaire provided to them. This pre-designed, pre-tested and structured questionnaire included topics relating to awareness about menstruation; source of information regarding menstruation, hygiene practiced during menstruation and restricted activities practiced during menstruation. At the end of the study, after collection of the questionnaire from the students, all their queries were answered satisfactorily by the research worker.

\section{Statistical analysis}

Data obtained were collated and analyzed statistically by simple proportions.

\section{RESULTS}

This study shows that the age of menstruating girls ranged from 14 to 17 years, maximum number of girls being between 14 and 15 years of age group. Among 350 respondents in the present study, $263(75 \%)$ were Muslims, whereas only twenty five $(25 \%)$ girls were Hindus. Fathers of most of the girls were labourers $(46.75 \%)$, followed by businessmen $(30 \%)$, service holders $(14.5 \%)$ and vendors $(8.75 \%)$. Mothers of most of the respondents were housewives $(98.75 \%)$.

\section{Knowledge and attitude}

In the present study, the mean age of menarche of the respondents was 13.2 years.

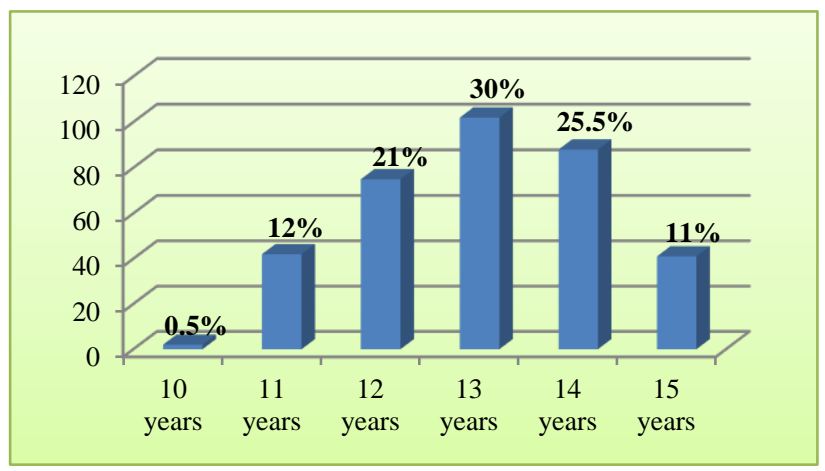

Figure 1: Age of menarche.

The study also depicts the status of mother's education; around $46 \%$ were illiterate, $27 \%$ had primary education, $12 \%$ had secondary education and $15 \%$ had higher education (Figure 2). 


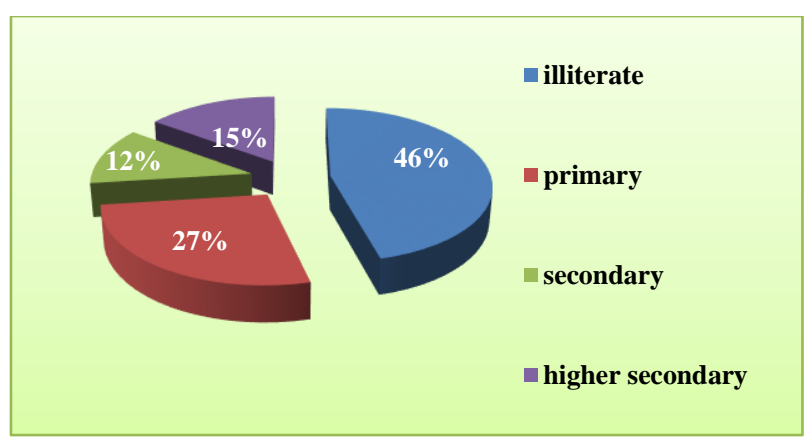

Figure 2: Depicts mother's education status and its effect.

The study done shows that the source of information about menstruation were $(70 \%)$ from mother, $(16 \%)$ from sisters, $(10 \%)$ from friends, $(2 \%)$ from teachers and $(2 \%)$ was from television and radio.

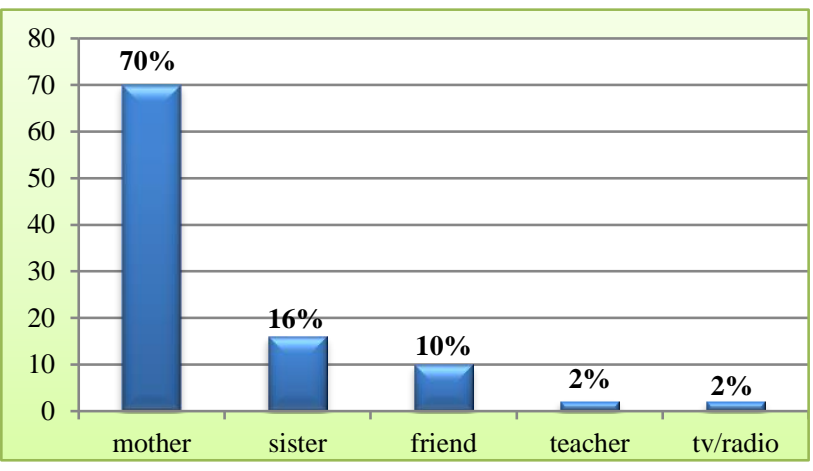

Figure 3: Source of information about menstruation.

The above mentioned study also shows the different beliefs and conception about menstruation among the respondents. It was observed that 130 (37\%) girls believed it as a physiological process. 77 (22\%) girls believed it as a curse of God. Most of the girls (41\%) did not know about the source of menstrual bleeding. More than half of the girls $(51.25 \%)$ were ignorant about the use of sanitary pads during menstruation.

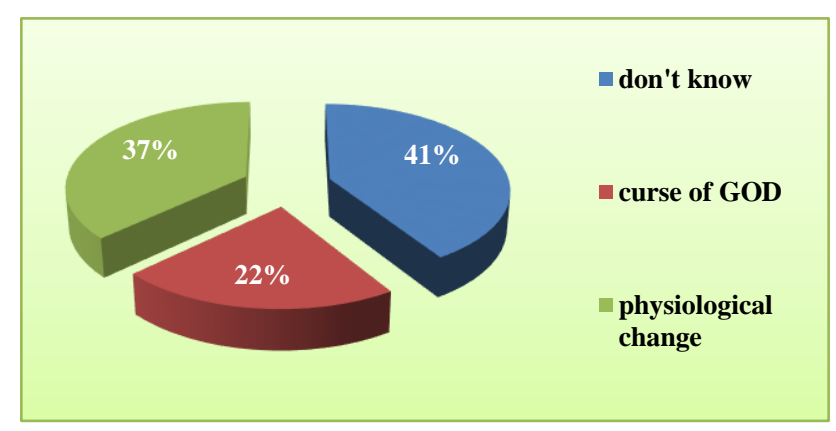

Figure 4: Knowledge about causes of menstruation.

\section{Pattern and problems during menstruation}

From the study done we have found that the duration of menstruation among adolescent girls. About 259 (>74\%) girls have periods between 5-7 days, 49 (14\%) have between 3-4 days, 18 (5\%) have for more than 7 days and $7 \%$ have for less than 2 days.

Table 1: Duration and length of menstrual cycle in adolescent girls.

\begin{tabular}{|l|l|}
\hline Duration of menstruation & $\%$ of girls \\
\hline$<2$ days & $74 \%$ \\
\hline $3-4$ days & $14 \%$ \\
\hline $5-7$ days & $7 \%$ \\
\hline$>7$ days & $5 \%$ \\
\hline Length of cycle & $67 \%$ \\
\hline$<28$ days & $19 \%$ \\
\hline 28-32 days & $14 \%$ \\
\hline$>32$ days & \\
\hline
\end{tabular}

It shows that around $52 \%$ of adolescent girls complains of dysmenorrhoea while $14 \%$ has irregular menses, $22 \%$ have backache and $10 \%$ excessive bleeding and only $2 \%$ complains of breast pain or tenderness.

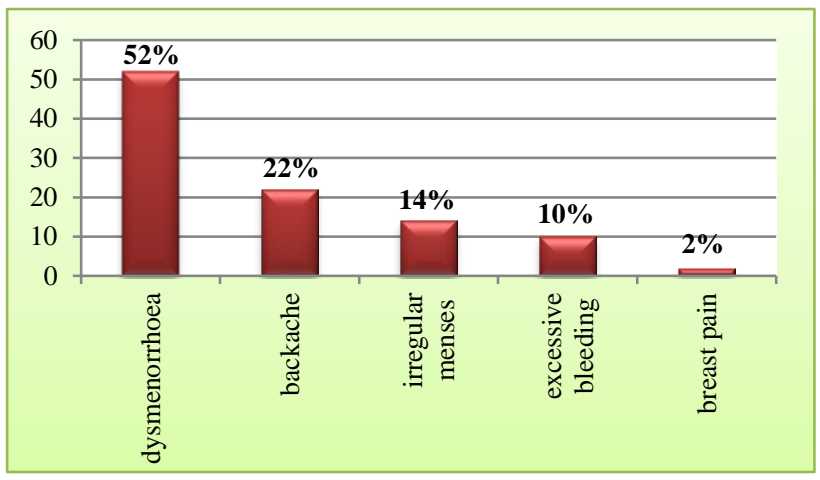

Figure 5: Problems faced by adolescent girls during menstruation.

\section{Seclusion and absenteeism}

About the culture and taboos we have found that $76 \%$ girls are not allowed to attend any religious acts or functions during menstruation, $22 \%$ are being restricted to have certain type of foods, $15 \%$ are being forced to sleep separately, and rest are not allowed to play or go to school.

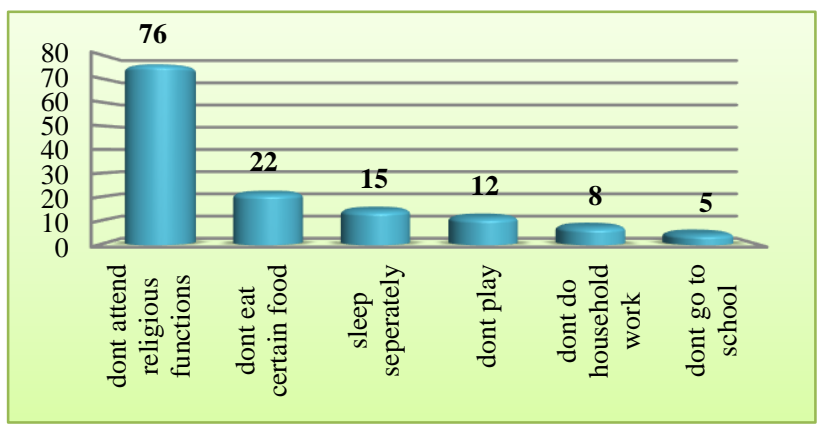

Figure 6: Restriction of daily activities during menstruation. 
Menstruation has been a wide cause of being absent from school. Around $24 \%$ girls remain absent from school during this period and (Figure 7) shows the cause of absenteeism. Maximum around 54\% amounting due to pain.

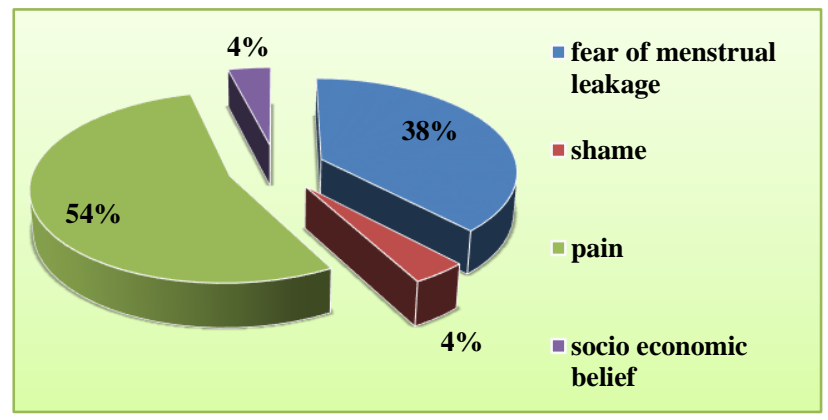

Figure 7: Cause of absenteeism from school.

\section{Hygiene related practices}

Regarding hygiene it has been found that in this era also cloth is still used as an absorbent during menses in $62 \%$ girls, only $22 \%$ girls use sanitary pads and rest $16 \%$ use old cloth.

Table 2: Type and number of absorbents used.

\begin{tabular}{|l|l|l|}
\hline $\begin{array}{l}\text { Type of } \\
\text { absorbent used }\end{array}$ & $\begin{array}{l}\% \text { of adolescent } \\
\text { using absorbent }\end{array}$ & $\begin{array}{l}\text { Absorbents } \\
\text { used per day }\end{array}$ \\
\hline New cloth & $62 \%$ & $4-5$ \\
\hline Sanitary pad & $22 \%$ & $2-3$ \\
\hline Old cloth & $16 \%$ & $4-5$ \\
\hline
\end{tabular}

Basically, reason found for not using sanitary pad is its unavailability (42\%), (24\%) mothers restriction, (16\%) due to shyness, $(12 \%)$ due to high cost and (4\%) due to lack of knowledge.

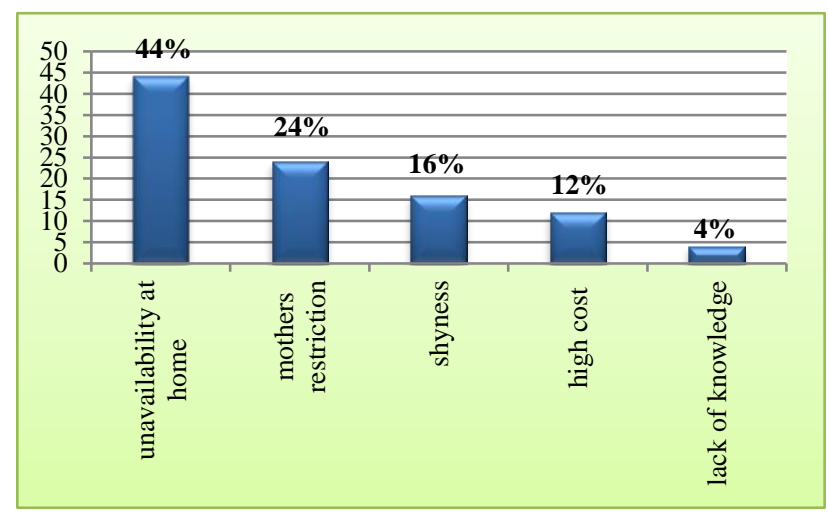

Figure 8: Reason for not using sanitary pad during menstruation.

\section{DISCUSSION}

In present study, the mean age at menarche was 13.2 years, which is similar to another study conducted in
Nagpur by Subhash et al while in study conducted by Keerti et al found 12.4 years is mean age of menarche. ${ }^{4}$ It is expected that the girls are provided correct information about menstruation before its onset, i.e., menarche. In present findings, it is seen that around $42 \%$ of school going adolescent girls was provided with the information before the onset of menstruation. A culture of silence surrounds menarche, an event which takes many young girls almost by surprise. The source of information was usually through school teachers and family relations especially mother, which is a good thing. But again, it was seen that the information provided was not adequate enough. The inadequate knowledge of mother was passed on to these young girls.

These findings are similar to other studies in Delhi where most of the young girls were previously unaware that it would happen and the information they are given is sparse. Similar findings are also seen from other parts of India like Varanasi, West Bengal, Andhra Pradesh and Rajasthan. ${ }^{1,3,7,8}$ These studies in India also found that mothers, sisters, and friends are usually the major source of information. ${ }^{9,10}$ Similar findings are also seen from studies in other parts of the world like Egypt, Pakistan, where it as seen that either inadequate or poor information was passed to the young girls through improper sources. 3,11

In present study, only $16 \%$ young women used reusable cloths (either alone or along with sanitary napkins). Obviously due to the poor socio-economic conditions of these families, they cannot afford to purchase costly sanitary napkins. But it is essential that the reused cloths should be properly washed but significant finding was $62 \%$ girls were using sanitary pad being residing in a city they had information regarding this. In similar study in Mumbai, it was found that $25 \%$ of young girls were still using reusable cloths. ${ }^{10}$ In study conducted in Andhra Pradesh $53.7 \%$ young girls use sanitary pad. ${ }^{1}$ In a study from Rajasthan and North India, the majority of the young girls were using and reusing old cloth, homemade napkins, and very few used cotton wool or sanitary napkins. $^{3,5}$ Cloth is the cheapest material used for protection during menstruation. All kinds of old, ragged, and rejected clothes are kept by women for this and used by the majority of women in the slum and in rural areas. 5,10 The main reasons for using homemade napkins were the inability to buy costly readymade sanitary napkins but also the lack of availability in rural areas.

Another important factor influencing their choice of sanitary protection methods was good absorbing capacity so there would not be any staining on the clothes. This question was, however, not elaborated in detail. Girls also need privacy and hygienic facilities at home as well as at school. Sanitary pads should be made available at schools for emergency use. Limited knowledge can result in many unhygienic practices like reusing same cloth again and again without proper washing, ignoring health problems, trying to manage the problems faced during 
menstruation on their own, etc. These adolescent and young girls frequently face problems and restrictions during the menstruation and these problems are usually not tackled in a proper manner.

We have found that about $66 \%$ of the girls were below the poverty line. We have also found that only $37 \%$ knew that it is a normal physiological process and only $10 \%$ are aware that it is coming from the genital tract.

In present study we had reported that among adolescent girls, dysmenorrhea in about $52 \%$ was the most common problem followed by pre-menstrual syndrome. Present findings are almost same. This affects the daily routines of majority of the girls leading to absenteeism from school/college and work. Studies from out of India also reported similar findings. A study from Egypt showed that the prevalence of menstrual problems and dysmenorrhea increased with age but very few had sought medical care because of menstrual problems. Also, in similar studies conducted in North India, South India and Mumbai similar findings and results were

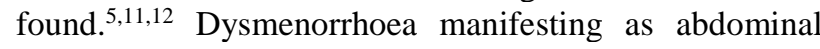
pain or discomfort has been found a Menstrual hygiene is one of the important risk factors for reproductive tract infections. ${ }^{12}$ Adolescent girls have been reported to suffer from various reproductive health problems associated with menstruation. Abdominal pain, bad odor of menstrual blood, burning during urination, and profuse discharge of menstrual blood were the most reported problems is the commonest problem in $52 \%$ of the girls. ${ }^{6}$

Practices of ritual seclusion particularly after the attainment of menarche like not attending religious functions was found in $76 \%$ of the girls. $24 \%$ girls in this study reported having been absent from school during menstruation mostly due to dysmenorrhoea. Fortunately, $96 \%$ know about sanitary pad but hardly $62 \%$ are using it. Reason for not it was mostly unavailability at home. Hardly $22 \%$ take bath daily during menstruation.

\section{CONCLUSION}

This study highlighted the need of adolescent girls to have accurate and adequate information about menstruation and its appropriate management \&healthy menstrual practices. Channels of communication such as mothers, sisters and friends, need to be emphasized for the delivery of such information. Mothers to be armed with the correct and appropriate information on reproductive health, so that she can give this knowledge to her growing girl child. It is also essential for the teachers, to impart reproductive health education, including menstrual hygiene to their students. Strong need to address issues like the restrictions which are imposed on or practiced by the girls. Considering lesser use of sanitary pads there is a need to mobilise girls to use sanitary pads. Education regarding reproductive health and hygiene should be included as a part of school curriculum. Need for the day is: open discussion and : Family involvement

This paper reemphasizes the important, urgent, and neglected need of providing correct knowledge to the entire community including adolescent and young girls. Correct knowledge will help them practice safe and hygienic menstrual practices and come out of traditional beliefs, misconceptions, and restrictions regarding menstruation. For this, the proper policies should be formulated and implemented, which can be part of overall health and community development policy. The physiology of the menstrual cycle, its connection to fertility, and the fact that menstruation is a normal process without any kinds of dirt should be part of family life/sexual education in schools. The health sector especially the public health system should play proactive role.

In order to eliminate needless limitations in relation to menstruation a number of changes are required in women's social and reproductive health situation, community planning in relation to sanitation and disposal, and product development and marketing at a low cost. If the economic stature of India is changing as a whole, women's situation with regard to menarche and menstruation may also change in the same direction.

Funding: No funding sources

Conflict of interest: None declared

Ethical approval: Not required

\section{REFERENCES}

1. Jogdand K, Yerpude P. A community based study on menstrual hygiene among adolescent girls. Indian $\mathbf{J}$ Maternal Child Health. 2011 Jul 1;13(3):1-6.

2. Dasgupta A, Sarkar M. Menstrual hygiene: how hygienic is the adolescent girl? Indian J Commun Med. 2008;33(2):77 -80.

3. Khanna A, Goyal RS, Bhavsar R. Menstrual practices and reproductive problems: a study of adolescent girls in Rajasthan. J Health Managm. 2005;7:91-107.

4. Mudey AB, Keshwani N, Mudey GA, Goyal RC. A cross-sectional study on the awareness regarding safe and hygienic practices amongst school going adolescent girls in the rural areas of Wardha district. Global J Health Sci. 2010;2(2):225-3.

5. Singh AJ. The place of menstruation in the reproductive lives of women of rural north India. Indian J Comm Med. 2006;31(1):10-14.

6. Ten $\mathrm{V}$ TA. Menstrual Hygiene: A neglected condition for the achievement of the Millennium Development Goals. Europe Ext Policy Advisors. 2007.

7. Kansal S, Singh S, Kumar A. Menstrual hygiene practices in context of schooling: A community study among rural adolescent girls in Varanasi. Indian journal of community medicine: official 
publication of Indian Assoc Preven Soc Medicine. 2016 Jan;41(1):39.

8. Prajapati J, Patel R. Menstrual hygiene among adolescent girls: A cross sectional study in urban community of Gandhinagar. JMR. 2015;1(4):122-5.

9. Yasmin S, Manna N, Mallik S, Ahmed A, Paria B. Menstrual hygiene among adolescent school students: An in-depth cross-sectional study in an urban community of West Bengal, India. IOSR J Dent Med Sci. 2013 Mar;5(6):22-6.

10. Thakur H, Aronnson A. Knowledge, practices, and restrictions related to menstruation among young women from low socioeconomic community in Mumbai, India. Front Public Health. 2014;2:72.

11. Ali TS, Rizvi SN. Menstrual knowledge and practices of female adolescents in urban Karachi, Pakistan. J Adolesc. 2010;33(4):531.
12. Narayana KA, Srinivasa DK, Petlo PJ. Puberty rituals, reproductive knowledge and health of adolescent school girls in south India. Asia Pacific Population Journal 2001;16(2):225 -38.

13. El-Gilany AH, Badawi K. Menstrual hygiene among adolescent schoolgirls in Mansoura, Egypt. Reprod Health Matters. 2005;13:147-52.

Cite this article as: Sultan S, Sahu DS. Knowledge, attitude and practices about menstruation and related problems in adolescent girls. Int J Reprod Contracept Obstet Gynecol 2017;6:5235-40. 